\title{
Isolement par partition bio guidé du principe actif myostimulant de l'extrait aqueux de Mareya micrantha (Benth) Mull. Arg. (Euphorbiaceae)
}

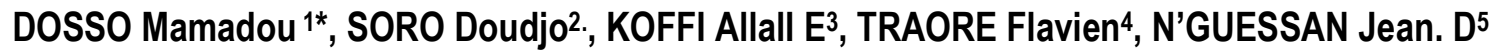 \\ 1 UFR Sciences Biologiques, Université Peleforo Gon Coulibaly, B.P 1328 Korhogo, Côte d'Ivoire. \\ ${ }^{2}$ Laboratoire des procédés industriels de synthèse de l'environnement et des énergies nouvelles, Institut National \\ Polytechnique Félix Houphouët-Boigny, B.P 1093 Yamoussoukro, Côte d'Ivoire \\ ${ }^{3}$ Laboratoire de Pharmacologie des substances naturelles, Université Lorougnon Guède, B.P 150 Daloa, Côte d'Ivoire \\ ${ }^{4}$ Laboratoire de Physiologie Animale, U.F.R. Biosciences, Université Félix Houphouët-Boigny 22 B.P. 582 Abidjan22, \\ Côte d'Ivoire. \\ ${ }^{5}$ Laboratoire pharmacodynamie Biochimie, Département de Biosciences, Université Félix Houphouët-Boigny de cocody \\ Abidjan, 22 BP 582 Abidjan 22, Côte d'Ivoire \\ Correspondant : DOSSO Mamadou coatcha1012@gmail.com Tél :(225) 58275953 / 84030802
}

Original submitted in on $5^{\text {th }}$ April 2017. Published online at www.m.elewa.org on $30^{\text {th }}$ June 2017 https://dx.doi.org/10.4314/jab.v114i1.7

\section{RÉSUMÉ}

Objectif: Isoler le principe actif myostimulant de l'extrait aqueux de Mareya micrantha (MAR) lors de sa partition dans différents solvants (70\% éthanol / eau ; $50 \%$ cyclohexane / eau et $50 \%$ acétate d'éthyle / eau), en utilisant le duodénum isolé de lapin comme marqueur de l'activité pharmacologique, afin d'identifier la fraction possédant la meilleure activité myostimulante.

Méthodologie et résultats : le dispositif d'enregistrement de l'activité mécanique du duodénum isolé de lapin, a permis d'évaluer les propriétés pharmacologiques de MAR, des fractions ((hydro-alcoolique) F1, (cyclohexanique) F2, (aqueuse) F3, (acétate d'éthyle) F4 et (aqueuse) F5) et précipité (P) obtenus par partition de MAR dans différents solvants ( $70 \%$ éthanol / eau ; $50 \%$ cyclohexane / eau et $50 \%$ acétate d'éthyle / eau). Les effets myostimulants de MAR diminuent fortement en milieu physiologique sans calcium et contenant de I'EDTA. Les fractions, hydro-alcoolique (F1), aqueuses (F3 ; F5), et le précipité (P) obtenus par partition de MAR, stimulent l'activité contractile du duodénum de lapin. La fraction acétate d'éthyle (F4) est myorelaxante, La F2 (cyclo-hexanique) est sans effet. L'étude dose réponse de F5 à des concentrations allant de 20 à 240 $\mu \mathrm{g} . \mathrm{mL}^{-1}$, montre qu'elle est $120 \%$ plus spasmogène que MAR. Ces effets inotropes positifs de F5 sont inhibés par l'atropine.

Conclusion et application: Les principes actifs myostimulants de MAR augmenteraient l'amplitude des contractions du duodénum isolé de lapin en mobilisant principalement le calcium extracellulaire puis intracellulaire comme en témoigne le résultat de l'expérience avec l'EDTA. La forte activité spasmogène de la F5 et l'expérience avec l'atropine, suggèrent que la partition de MAR a enrichi la F5 en principe actifs myostimulants de nature cholinergique. Ces données indiquent que la partition peut contribuer à isoler de manière significative les principes actifs des plantes médicinales, y compris ceux de MAR aux propriétés myostimulantes, justifiant son utilisation comme laxatif.

Mots clés : Mareya micrantha, fractions, myostimulant, Atropine 


\section{ABSTRACT}

Objective: To isolate the myostimulant active principle in Mareya micrantha (MAR) aqueous extract during its partition in different solvents (70\% aqueous-ethanol, 50\% aqueous-cyclohexane and $50 \%$ aqueous ethylacetate), while using the isolated rabbit duodenum as a pharmacological activity marker in order to identify the fraction having the best myostimulant activity.

Methodology and results : The mechanical activity recording device of the isolated rabbit duodenum, allowed the assessment of the pharmacological properties of MAR, ((Hydro-alcoholic) F1, (cyclohexane) F2, (aqueous) F3, (ethylacetate) F4, (aqueous) F5) fractions and the precipitate (P) obtained by MAR partition in different solvents (70\% aqueous-ethanol, $50 \%$ aqueous-cyclohexane and $50 \%$ aqueous-ethylacetate). The myostimulant effects of MAR decrease strongly in physiological environment without calcium and in presence of the EDTA. The Hydro-alcoholic (F1) and aqueous fractions (F3; F5), and the precipitate (P) stimulate the rabbit duodenum contractile activity. The ethylacetate fraction (F4) is myorelaxant; the cyclohexane fraction (F2) has no effect. The Dose-response study of $\mathrm{F} 5$ with concentrations ranging from 20 to $240-\mu \mathrm{g}$. $\mathrm{ml}^{-1}$, shows that it is $120 \%$ more spasmogen than MAR. These inotropic positive effects of aqueous fraction (F5) are inhibited by atropine.

Conclusion and application: The myostimulant active principles of MAR increased the amplitude of the contractions of the isolated rabbit duodenum by mainly mobilizing the extracellular calcium then the intracellular calcium as with EDTA. The high spasmogen activity of the aqueous fraction (F5) and the experiences with atropine suggest that this partition of MAR enriched the F5 in myostimulant active principles of cholinergic nature. These data indicate that the partition can significantly contribute to isolate the active principles from MAR with myostimulant properties, justifying its use as laxative.

Keywords: Mareya micrantha, fractions, myostimulant, Atropine

\section{INTRODUCTION}

Mareya micrantha est un arbuste pouvant atteindre 4 mètres de hauteur présentant des feuilles simples, oblongues, galbes, généralement alternes et pubescentes à la face inférieure, puis disposées en forme de rameau. Elle est utilisée traditionnellement par les peuples de Côte d'Ivoire pour ses propriétés laxatives et ocytociques (MAC FOY et CLINE, 1986 ; GUEDE-GUINA et al., 1995). En Sierra Leone, la décoction des feuilles sèches sert à déparasiter les enfants, une infusion des feuilles est utilisée pour soulager les maux de tête et les maux d'estomac. Ses feuilles et Ses tiges sont censées guérir la trypanosomiase et la leishmaniose (DJAMA et al., 2011). Des études pharmacologiques de Mareya micrantha indiquent qu'une macération éthanoïque et aqueuse des feuilles possède une activité antibactérienne contre Entérobacter aérogènes, Agrobactérium tumefaciens, Bacille subtillis, Clostridium Sporogenes, Escherichia coli (MAC FOY et CLINE, 1990). Cette plante a des effets myostimulants sur l'iléon du cochon d'inde (TSAl et al., 1995), et le duodénum isolé de lapin (TRAORE et al., 2004). En plus de l'activité cardio-dépressive de l'extrait aqueux de Mareya micrantha observée chez les mammifères (ABO, 1996), l'extrait aqueux de cette essence naturelle améliore le transit intestinal chez le rat (MEITE et al., 2010). Aussi, le mélange des huiles essentielles de Mareya micrantha, Mitracarpus scaber et Cassia alata contient des agents antifongiques ; le savon de ce mélange permet le traitement de psoriasis (THES et al, 2006 ; THES et al., 2011 ; SOUMAHORO et al., 2014). A la dose de $100 \mathrm{mg} / \mathrm{Kg}$ de poids corporel Mareya micrantha, a des effets cardio-protecteurs chez le lapin, selon DOUMBIA et al., (2014). Les données toxicologiques et phytochimiques, attestent que l'extrait aqueux de Mareya micrantha est peu toxique et contient des alcaloïdes des polyphénols des saponososides et des flavonoïdes (Dosso et al., 2012). Toutes ces études donnent cependant peu d'informations sur les fractions de l'extrait aqueux de Mareya micrantha. Pour pallier ces insuffisances de données sur les fractions de cette plante, il s'est agi dans cette étude d'isoler le principe actif myostimulant de l'extrait aqueux de Mareya micrantha lors de sa partition dans différents 

l'extrait aqueux de Mareya micrantha (Benth) Mull. Arg. (Euphorbiaceae)

solvants, en utilisant le duodénum isolé de lapin comme marqueur de l'activité pharmacologique afin

\section{MATÉRIEL ET MÉTHODES}

Matériel végétal: Les feuilles fraiches de Mareya micrantha ont été récoltées dans la localité d'Akoupé, en Juin 2007, identifiées et authentifiées par Pr Ake Assi Laurent du département de botanique de l'Université Félix Houphouët Boigny (Côte d'Ilvoire). Après identification, un spécimen a été déposé sous le numéro 1804 à l'herberie du Centre National de Floristique de l'Université Félix Houphouët-Boigny.

Matériel animal: Pour nos expériences pharmacologiques, nous avons utilisé des lapins de l'espèce Oryctolagus cuniculus (Léporidés). Ces lapins proviennent de la commune d'Abobo situé à la banlieue d'Abidjan, capitale économique de la République de Côte d'Ivoire. Ils pèsent en moyenne $2 \mathrm{Kg}$. Ils ont été acclimatés dans les conditions ambiantes $\left(28^{\circ} \pm 3^{\circ} \mathrm{C}\right)$ pendant une semaine à l'animalerie de l'unité de formation et de recherches (UFR) Biosciences de l'Université Félix Houphouët-Boigny de Cocody avant d'être utilisés pour les expériences. La photopériode est de 12/24 heures.

Dispositif expérimental : Le dispositif expérimental comporte un bain-marie thermostaté, d'une cuve à organe isolé rempli de solution physiologique de type Mac Ewen, contenu dans des flacons placés à $40 \mathrm{~cm}$ de l'appareillage. Les liquides contenus dans les flacons dans des cathéters de polyvinyle, puis des serpentins qui permettent à ces solutions de se réchauffer à une température de $38 \mathrm{C}$. Cette arrivée de liquide est contrôlée par un robinet de sélection à voies multiples. La cuve à organe isolé peut être vidée grâce à une purge située au bas de l'appareillage.

\section{Méthodes}

Méthode de préparation de l'extrait aqueux: Les feuilles de Mareya micrantha sont séchées à l'abri du soleil, puis réduites en poudre. 80 grammes de cette poudre sont dissous dans 2 litres d'eau distillée. La mixture est macérée pendant 24 heures à l'aide d'un agitateur magnétique (AGIMATIC-N) puis filtrée sur du coton hydrophile. Le filtrat est évaporé à l'aide d'un évaporateur rotatif de marque Buchi à $70^{\circ} \mathrm{C}$. Après évaporation totale, on obtient une poudre dont une partie est utilisée comme extrait aqueux (MAR) et l'autre est fractionnée.

Méthode de fractionnement par partition de l'extrait aqueux : L'extrait aqueux de Mareya micrantha (1,5 g) est dissous dans un litre d'éthanol à $70 \%$ (Ethanol/eau ; $70 / 30 ; \mathrm{v} / \mathrm{v})$. Le mélange est agité pendant 6 heures et d'identifier la fraction possédant la meilleure activité myostimulante.

laissé au repos dans un ballon à décanter pendant 24 heures. Un surnageant hydro-alcoolique (F1) et un précipité $(\mathbf{P})$ ont été obtenus, recueillis séparément puis évaporés à l'aide d'un évaporateur rotatif de marque Buchi à $60^{\circ} \mathrm{C}$. Les culots obtenus sont séchés à l'étuve à $50^{\circ} \mathrm{C}$. Une partie de $\mathrm{F} 1$ reprise avec un mélange de cyclohexane-eau $(5: 5 ; \mathrm{v} / \mathrm{v})$ est soumise au même processus. On obtient une fraction cyclo-hexanique (F2) et une fraction aqueuse (F3). L'extrait obtenu à partir de la phase aqueuse (F3) est repris dans un mélange acétate d'éthyle-eau $(5: 5 ; \mathrm{v} / \mathrm{v})$. La solution subit le même traitement que les autres mélanges. Sont obtenus une fraction acétate d'éthyle (F4) et une fraction aqueuse (F5).

Prélèvement et mise en place de l'organe isolé : Le lapin mis à jeun pendant 24 heures, est assommé. A la suite d'une laparotomie médiane, des segments de duodénum long de $3 \mathrm{~cm}$ sont immédiatement prélevés et maintenus en vie dans une solution de Mac Ewen glucosé et oxygéné. Un des fragments est monté sur l'appareil d'enregistrement dans un godet contenant 250 ml de Mac Ewen en (mM) Nacl $130 ; \mathrm{Kcl} 2,5 ; \mathrm{Cacl} 22,40$; $\mathrm{NaH} 2 \mathrm{PO} 31,18$; NaHCO3 11,90; MgCl2 0,24; glucose 2,2 de $\mathrm{PH} 7,4$ continuellement oxygéné et de température $38 \mathrm{C}$.

A l'aide d'un fil passé à travers la paroi du fragment de duodénum on réalise un nœud à une extrémité du fragment de duodénum permettant de l'accrocher à l'intérieur de la cuve à organe isolé. L'autre extrémité est reliée par un autre fil au stylet dont la plume est en contact d'un papier enduit de fumée noire, enroulé sur un cylindre soumis à une rotation de vitesse constante.

Protocole expérimentale: A l'aide d'une seringue graduée, un volume des dilutions réalisées à partir des substances tests est introduites dans la cuve à organe isolé, un godet contenant $250 \mathrm{ml}$ de Mac Ewen.

Pour passer d'un test au suivant la préparation est lavée soigneusement avec du Mac Ewen (750 ml) afin d'éviter l'effet cumulatif des substances tests.

Tri phytochimique : Les tests d'analyse standard (SILVA et al., 1998) ont été utilisés pour la recherche des alcaloïdes, des flavonoïdes, des tanins, des composés phénoliques et des saponines dans la fraction F5.

Analyse statistique: Les données statistiques exprimées en moyennes \pm erreur standard ( $M \pm S E M$ ) ont été obtenues à partir des $(n=4)$ expériences séparées. Les moyennes calculées ont été comparées à partir du 
test (t) de Student. Lorsque $p \leq 0,05$, la différence est dite significative. Les courbes et l'analyse statistique ont été

\section{RÉSULTATS}

Effet de MAR en milieu sans calcium : En milieu $0 \mathrm{Ca}^{2+}$ contenant de l'EDTA $3.10^{-2} \mathrm{mg} \cdot \mathrm{mL}^{-1}$, les contractions rythmiques du duodénum de lapin sont totalement abolies, l'ajout de MAR (160 $\left.\mu \mathrm{g} \cdot \mathrm{mL}^{-1}\right)$ à ce milieu provoque une légère contracture de $333,33 \pm 35 \mathrm{mg}$ de force (Figure 1A). La Figure 1B. présente La variation de la contraction du duodénum de lapin en milieu physiologique dépourvu de calcium et contenant de l'EDTA $(n=4)$.

Effet de MAR et de ses fractions sur les contractions rythmiques du duodénum de lapin: MAR et ses réalisées avec le logiciel Graph Pad Prism 5.01, San Diego, CA, USA.

fractions ( $\mathrm{F} 1, \mathrm{P}, \mathrm{F} 3$ et $\mathrm{F} 5)$ testés à $160 \mu \mathrm{g} . \mathrm{mL}^{-1}$, augmentent la contraction rythmique du duodénum de lapin respectivement de $1142,85 \pm 3(P<0,05) ; 428,57$ $\pm 4(P<0,05) ; 285,71 \pm 7(P<0,05) ; 785,71 \pm 6(P>$ $0,05)$ et $1371,42 \pm 8 \mathrm{mg}$ force $(P<0,01)$ (Figure $2 \mathrm{~A})$. F4 en revanche diminue la contraction de $428,57 \pm 4 \mathrm{mg}$ force $(P<0,001)$. F2 n'entraine aucun effet. Ces expériences ont été réalisées quatre fois $(n=4)$. De toutes ces fractions, F5 (160 $\left.\mu \mathrm{g} \cdot \mathrm{ml}^{-1}\right)$ est celle qui présente un effet myostimulant supérieur (120\%) à celui de MAR (Figure 2B).

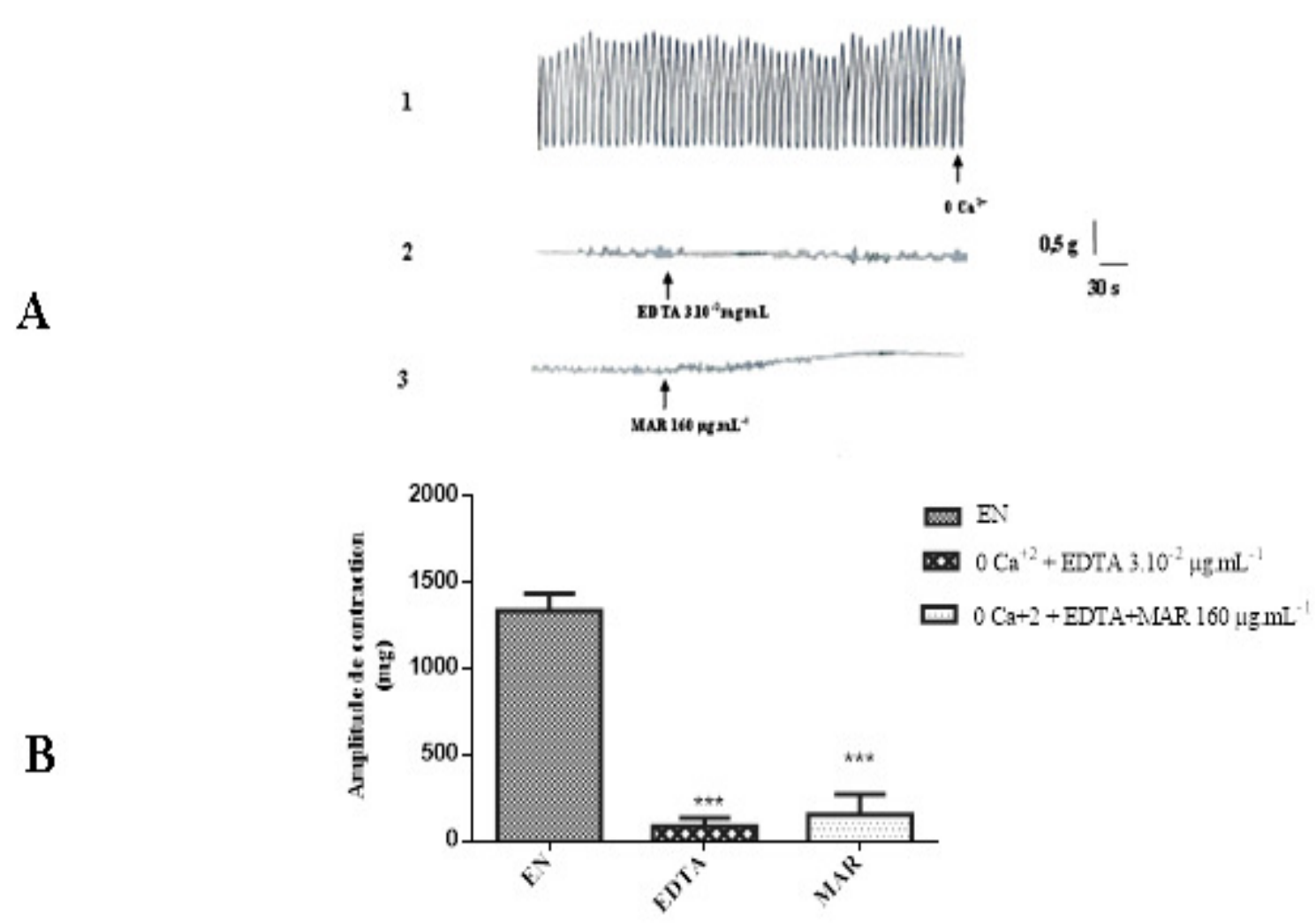

Figure 1 : Effet de l'extrait aqueux de Mareya micrantha sur l'activité contractile du duodénum de lapin en milieu physiologique dépourvu de calcium.

A Effet de MAR en milieu $\mathrm{Oca}^{2+}$

A- Enregistrement Normal (enregistrement témoin)

B- $\quad$ Effet de $0 \mathrm{Ca}^{2+}$ suivie de l'effet d'EDTA

C- $\quad$ Suite de 2, effet de $0 \mathrm{Ca}^{2+}$ EDTA suivie de l'effet de MAR $160 \mu \mathrm{g} \cdot \mathrm{mL}^{-1}$

B Augmentation de la contraction induite par MAR en milieu $0 \mathrm{ca}^{2+}$

En milieu physiologique dépourvu de calcium et en présence de l'EDTA, MAR provoque une contracture.

Ces valeurs expriment une augmentation de la contraction en fonction de la concentration de MAR en milieu $0 \mathrm{Ca}^{2+}$ 
A

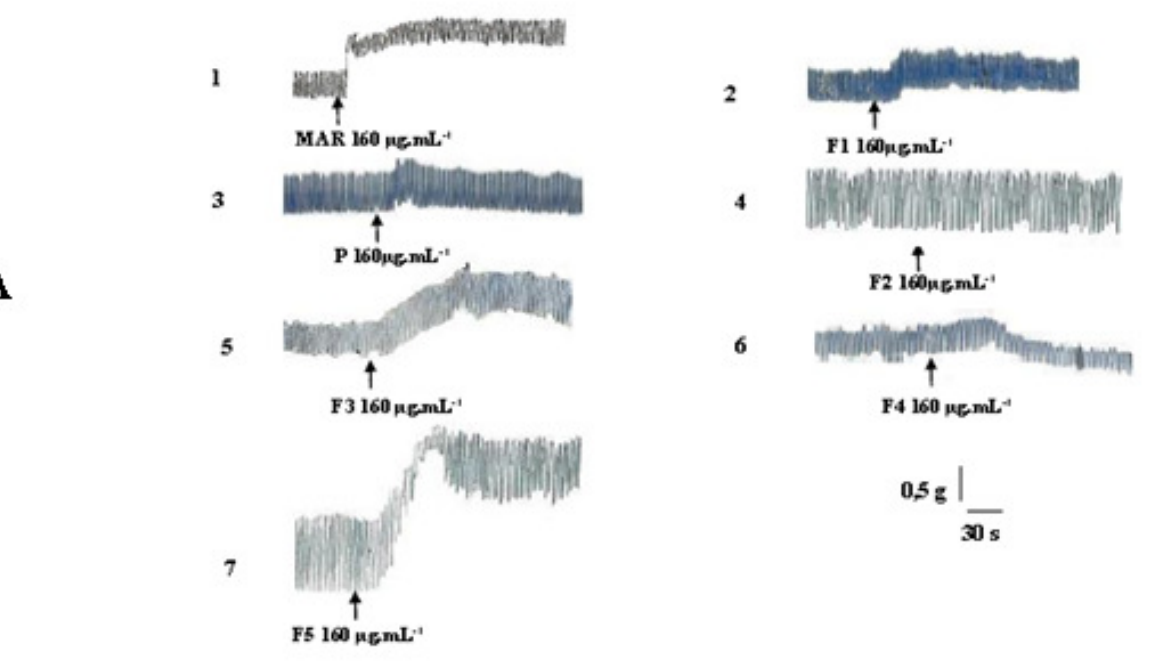

B

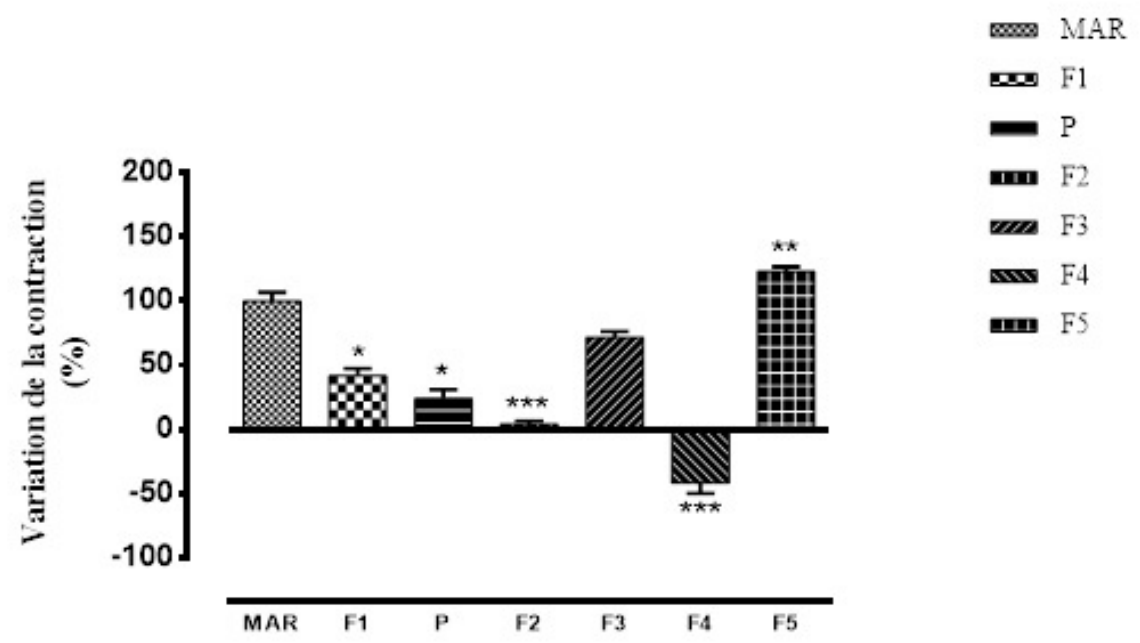

Figure 2: Effet d'un extrait aqueux de Mareya micrantha et de ses différentes fractions sur l'activité contractile du duodénum de lapin

\section{A : Effet de MAR et de ses fractions}

Effet de MAR $160 \mu \mathrm{g} \cdot \mathrm{mL}^{-1}(1)$; Effet de F1 $160 \mu \mathrm{g} \cdot \mathrm{mL}^{-1}(2)$; Effet de P $160 \mu \mathrm{g} \cdot \mathrm{mL}^{-1}(3)$; Effet de F2 $160 \mu \mathrm{g} \cdot \mathrm{mL}^{-1}$ (4) ; Effet de F3 160 $\mu \mathrm{g} \cdot \mathrm{mL}^{-1}$ (5) ; Effet de F4 $160 \mu \mathrm{g} \cdot \mathrm{mL}^{-1}$ (6) ; Effet de F5 $160 \mu \mathrm{g} \cdot \mathrm{mL}^{-1}$ (7)

$B$ : Variation des contractions induite par MAR et de ses fractions

F5 induit l'effet inotrope positif le plus important sur la contraction du duodénum

Ces valeurs expriment une augmentation de la contraction en fonction de la fraction testée à $160 \mu \mathrm{g} \cdot \mathrm{ml}^{-1}$ (Moyenne $S E M,{ }^{* *} P<0,01$, $n=4)$.

Effet dose-réponse de F5 sur les contractions rythmiques du duodénum de lapin: Pour des concentrations comprises entre 20 et $240 \mu \mathrm{g} \cdot \mathrm{mL}^{-1}$, F5 provoque une augmentation dose-dépendante de la contraction du duodénum de lapin (Figure 3A). Pour un intervalle de concentration allant de $20 \mu \mathrm{g} \cdot \mathrm{mL}^{-1}$ à 40 $\mu \mathrm{g} . \mathrm{mL}^{-1}, \mathrm{~F} 5$ induit une augmentation non significative $(P>$ $0,05)$ de la contraction. Par contre au-delà de ces concentrations, F5 provoque une augmentation significative de la contraction de $1500 \mathrm{mg}(75 \%)$ à 2000 $\mathrm{mg}$ force $(100 \%)(P<0,01-0,001)$. À partir de $40 \mu \mathrm{g} . \mathrm{mL}-$ 1 , on enregistre une augmentation du tonus de base, toutefois la fréquence des contractions reste constante. La courbe dose réponse de $\mathrm{F} 5$ est une sigmoïde avec une $\mathrm{EC}_{50}$ de $63,63 \pm 3 \mu \mathrm{g} \cdot \mathrm{mL}^{-1}$ (Figure 3B). 
Effet de F5 en présence d'ATR sur les contractions rythmiques du duodénum de lapin :La Figure 4A représente un enregistrement type de $\mathrm{F} 5$ en présence d'ATR sur l'activité contractile du duodénum de lapin. En présence d'ATR à $1,15.10^{-7} \mu \mathrm{g} \cdot \mathrm{ml}^{-1}, \mathrm{~F} 5$ de 40 à 80 $\mu \mathrm{g} \cdot \mathrm{mL}^{-1}$ provoque une augmentation faible et transitoire de la contraction qui varie de $642,85 \pm 8$ à $700,28 \pm 7$ $\mathrm{mg}$ force $(P>0,05)$. Ces augmentations s'accompagnent de celles du tonus de base qui retournent à la normale après 3 minutes. En présence d'ATR à 1,15.10-7 $\mu \mathrm{g} \cdot \mathrm{ml}^{-1}$, l'augmentation de la contraction induite par F5 de 160 $\mu \mathrm{g} \cdot \mathrm{ml}^{-1}$ à $240 \mu \mathrm{g} \cdot \mathrm{mL}^{-1}$ est fortement réduite $(P<0,05-$ $0,01)$. La courbe de la variation moyenne $(n=4)$ de la contraction du duodénum de lapin en fonction de la concentration de MAR en présence d'ATR à 1,15.10-7 $\mu \mathrm{g} \cdot \mathrm{ml}^{-1}$ est une sigmoïde avec une $\mathrm{EC}_{50}$ de 316,43 \pm 2

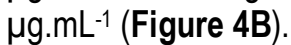

Tri phytochimique de F5: La F5 contient des alcaloïdes, des flavonoïdes, des quinones, des tanins, des polyphénols et des saponosides.

A

$\mathbf{1}$

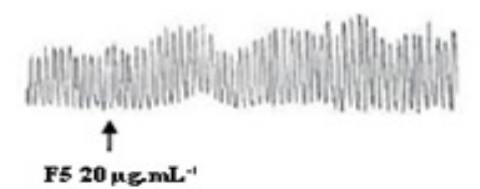

s.

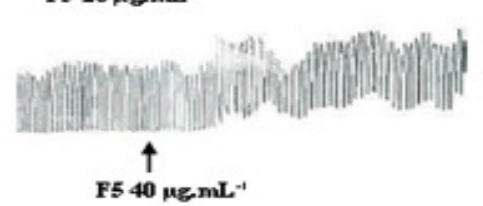

$0,\left.5 \mathrm{~g}\right|_{\frac{30}{30}}$

B

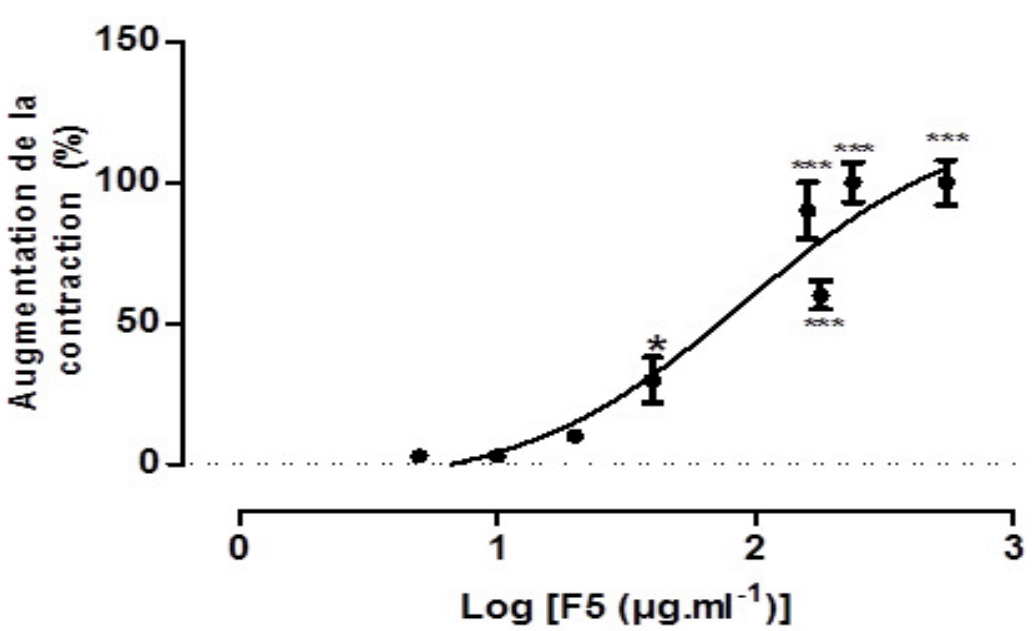

Figure 3 : Effet dose réponse de la fraction F5 sur l'activité contractile du duodénum de lapin A : Effet dose-réponse de F5

Effet de la fraction F5 à $20 \mu \mathrm{g} \cdot \mathrm{mL}^{-1}$ (1) ; $40 \mu \mathrm{g} \cdot \mathrm{mL}^{-1}$ (2) ; $80 \mu \mathrm{g} \cdot \mathrm{mL}^{-1}$ (3) ; $160 \mu \mathrm{g} \cdot \mathrm{mL}^{-1}$ (4) ; $240 \mu \mathrm{g} \cdot \mathrm{mL}^{-1}(5)$

$B$ : Augmentation des contractions en fonction de la concentration de F5

F5 provoque l'augmentation dose-dépendante des contractions du duodénum de lapin

Les valeurs expriment une augmentation de la contraction du duodénum de lapin en fonction de la concentration de F6

(Moyenne \pm SEM, ${ }^{*} P<0,05 ;{ }^{* * *} P<0,001 ; n=4$ ). 
A
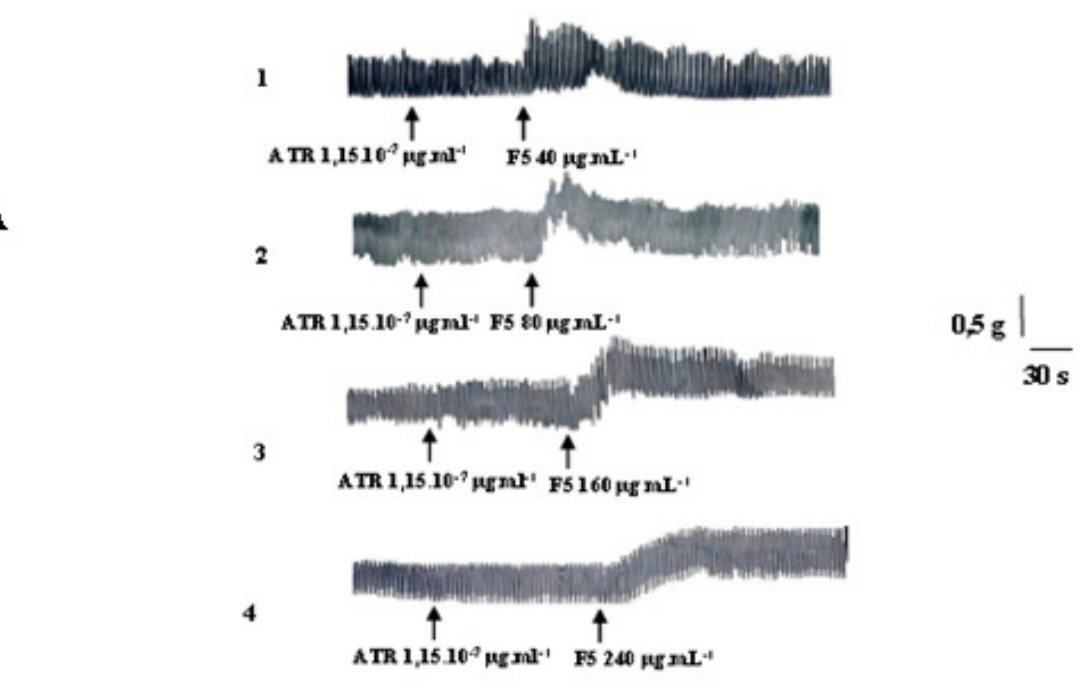

B

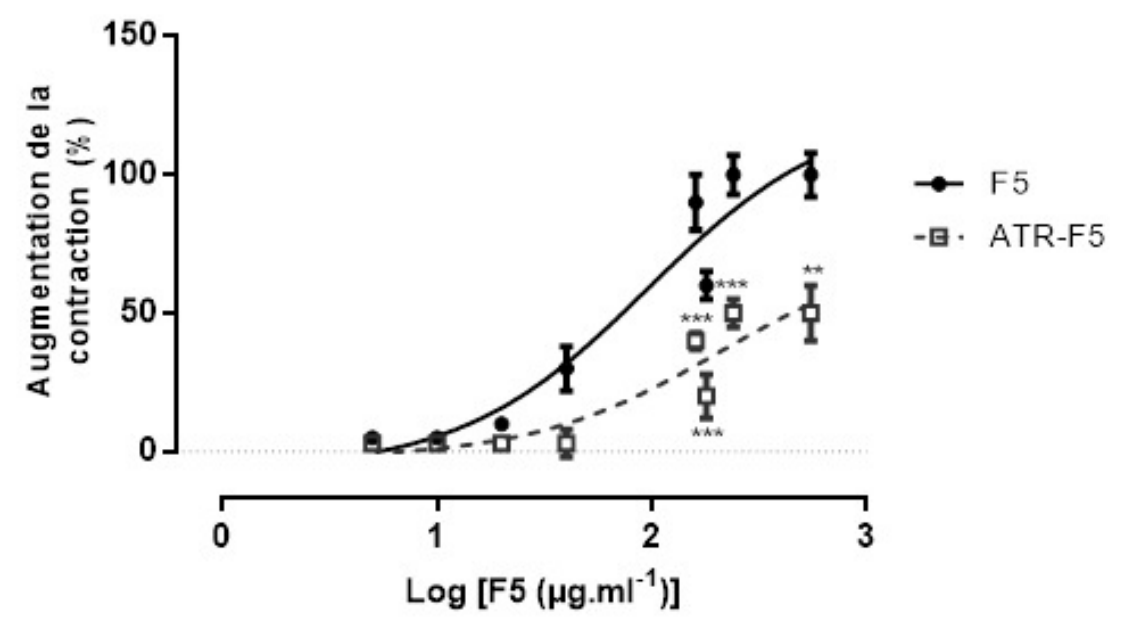

Figure 4 : Effet de la fraction F5 en présence d'ATR sur l'activité contractile du duodénum de lapin $A$ : Effet de la fraction $\mathrm{F} 5$ en présence d'ATR

1- $\quad$ Effet d'ATR 1,15.10-7 $\mu \mathrm{g} \cdot \mathrm{ml}^{-1}$ (1ère flèche) suivie de l'effet de F5 $40 \mu \mathrm{g} \cdot \mathrm{mL}^{-1}$ (2ème flèche)

2- $\quad$ Effet d'ATR 1,15.10-7 $\mu \mathrm{g} \cdot \mathrm{ml}^{-1}$ (1ère flèche) suivie de l'effet de F5 $80 \mu \mathrm{g} \cdot \mathrm{mL}^{-1}$ (2ème flèche)

3- $\quad$ Effet d'ATR 1,15.10-7 $\mu \mathrm{g} \cdot \mathrm{ml}^{-1}$ (1ère flèche) suivie de l'effet de F5 $160 \mu \mathrm{g} \cdot \mathrm{mL}^{-1}$ (2ème flèche)

4- Effet d'ATR 1,15.10-7 $\mu \mathrm{g} \cdot \mathrm{ml}^{-1}$ (1ère flèche) suivie de l'effet de F5 $160 \mu \mathrm{g} \cdot \mathrm{mL}^{-1}$ (2ème flèche)

$B$ : Augmentation des contractions induites par la fraction F5 en présence d'ATR

Les valeurs expriment une augmentation de la contraction du duodénum de lapin en fonction de la concentration de F5 en présence d'ATR 1,15.10-7 $\mu \mathrm{g} \cdot \mathrm{ml}^{-1}$ (Moyenne \pm SEM, ${ }^{* *} P<0,01 ;{ }^{* * *} P<0,001 ; n=4$ )

ATR inhibe l'augmentation de la contraction du duodénum provoquée par F5.

\section{DISCUSSION}

L'extrait aqueux de Mareya micrantha (MAR), contient des substances myostimulantes de types cholinergiques et histaminergiques, ces substances bioactives consécutivement à la stimulation des récepteurs correspondants, mobiliseraient le calcium externe puis interne pour augmenter l'amplitude des contractions du duodénum isolé de lapin (Traore et al., 2004 ; Dosso et al., 2012). La perte des effets myostimulants de l'extrait aqueux de Mareya micrantha dans le milieu physiologique sans calcium et en présence de l'EDTA, laisse entrevoir que ces principes actifs myostimulants sont principalement liés au calcium extracellulaires dont 

l'extrait aqueux de Mareya micrantha (Benth) Mull. Arg. (Euphorbiaceae)

l'entrée provoquerait secondairement la libération du calcium interne. Ces résultats rappellent ceux de Sesamum radiatum (Pedaliaceae) (KONAN et al., 2011). Les fractions obtenues par partition de MAR dans des solvants différents $(70 \%$ éthanol / eau; $50 \%$ cyclohexane / eau et $50 \%$ acétate d'éthyle / eau) et testées à $160 \mu \mathrm{g} \cdot \mathrm{ml}^{-1}$ sur le modèle du duodénum isolé de lapin, montrent que MAR, les fractions (F1, F3 et F5) et le précipité $(P)$ stimulent l'activité contractile du duodénum isolé de lapin, contrairement à F4 qui a un effet myorelaxant sur le même organe. Quant à F2 avec la même concentration, elle est sans effet. Ces effets pharmacologiques divers et quelquefois antagonistes des fractions de MAR, sont similaires aux effets des fractions d'autres plantes utilisées en pharmacopée africaine. $\mathrm{Ce}$ sont Anogeissus leiocarpus (ELOFF, 2011) et Polygonum minus (QADER et al., 2012). Pour des concentrations comprises entre 20 et $240 \mu \mathrm{g}$. mL-1 la fraction F5 la plus spasmogène de toutes ces fractions, présente un profil

\section{CONCLUSION GÉNÉRALE ET PERSPECTIVE}

Le fractionnement par partition de l'extrait aqueux de Mareya micrantha a abouti à l'isolement partiel des principes actifs myostimulants contenus dans F5. Pour augmenter l'amplitude des contractions du duodénum de lapin, ces principes actifs tels ceux de MAR mobiliseraient principalement le calcium extracellulaire puis

\section{RÉFÉRENCES BIBLIOGRAPHIQUES}

Abo J. C. (1996) Effets pharmacologiques d'un extrait aqueux de Mareya micrantha sur l'activité cardiovasculaire de mammifères. Thèse 3ème cycle, Université d'Abidjan, 144 pages.

Djama A.A. D., Kouassi G.M.C., Koua A.A., Osfosu F.G. et Aboh I.J. K. (2011) Trace Elements Analysis of Some Antiparasitic Medicinal Plants in Côte d'Ivoire Using Energy-Dispersive X-ray Fluorescence (EDXRF) Technique. Curr. Res. J. Biol. Sci, 3(3): 209-215

Dosso M., Méité S., Yéo D., Traoré F., Djaman A. et N'Guessan J.D. (2012) cholinergic and histaminergic of activities of aqueous extract of Mareya micrantha (Benth) Mull. Arg. (Euphorbiaceae)

Doumbia I., Ouattara K., Coulibaly A. F., Yapi H. F., Djaman A. J. et N'guessan J. D. (2014) Évaluation de l'effet cardioprotecteur de l'extrait aqueux de Mareya micrantha (Euphorbiaceae) chez le lapin

Eloff A. (2011) In vitro anthelmintic effect of Anogeissus Leiocarpus (DC) Guill \& Perr. Leaf extracts and myostimulant dose-dépendant. Elle est $120 \%$ plus efficace que MAR, on en déduit que le fractionnement a permis d'isoler au moins en partie les substances myostimulantes contenues dans F5. Comme l'extrait aqueux de Mareya micrantha (TRAORE et al., 2004), les effets myostimulants de F5 diminuent partiellement en présence de ATR à 1,15.10-7 $\mu \mathrm{g} \cdot \mathrm{ml}^{-1}$, suggérant ainsi qu'elle agirait en partie selon le même mécanisme que celui-ci. Autrement, les principes actifs de la fraction F5 augmenteraient l'amplitude de contraction du duodénum de lapin en mobilisant le calcium externe puis interne, via la stimulation des récepteurs cholinergiques. Aussi, le tri phytochimique de F5 montre qu'elle contient des alcaloïdes, des polyphénols, des flavonoïdes, des saponines et des tanins. Ces molécules d'intérêts pharmacologiques peuvent expliquer les propriétés myostimulantes de F5, et justifier l'utilisation traditionnelle de Mareya micrantha comme laxatif.

intracellulaire suite à la stimulation des récepteurs cholinergiques, d'où son effet laxatif. Le tri phytochimique de la F5 indique cependant qu'elle contient toujours plusieurs groupes de molécules, il convient alors de réaliser un second fractionnement afin d'identifier les molécules responsables de son effet myostimulant.

fractions on developmental stages of Hemonchus Contortus. Afr J Trad CAM, 8(2): 134-139.

Guede-Guina F., Tsai C.S., Smith M.O., Vangah M.M., Benny W. et Ochillo R.F. (1995) The use of isolated functional heart to pharmacologically characterize active ingredients in the aqueous extracts of Mareya micrantha. J. Ethnopharmacol, 45: 19-26.

Konan B.A., Amonkan A.K., Ahui M.L.B., Bouafou G.M.K. (2011) Myostimulating effect of Sesamum radiatum aqueous leaf extract in isolated guineapig Taenia caeci contractile activity. Afr J Trad CAM, 8 (4): 377-385.

Mac foy C.A. et Cline E.I. (1990) Somme medical plants used against bacterial infection of mans in Gloucester village (Sierra Leone). J. the Pharmaceutical Society of Sierra Leone, 1: 3949.

Meite S., Calixte B., Dodehe Y., Jacques Y.D., Joseph A.D. et David J.N. (2010) laxative activities of Mareya micrantha (Benth.) Müll. Arg. 
(Euphorbiaceae) leaf aqueous extract in rats. BioMed Central, $10: 7$

Qader S W., Abdulla M A., Chua L.S., Sirat H.M. et Hamdan S. (2012) Pharmacological Mechanisms Underlying Gastroprotective Activities of the Fractions Obtained from Polygonum minus in Sprague Dawley Rats. Int J Mol Sci, 13: 1481-1496.

Silva GL., Lee I. Kinghom AD : Special problems with the extraction of plants. Methods in Biotechnology (Natural product isolate) New Jersey : Humana pressCamell RJP 1998, 343-383.

Soumahoro Thes Pehie M., Bagre I, Doumbia I et Djama Allico J. (2014) Action soap misca-mates in the treatment of psoriasis. The Experiment, Vol.24 (3) $1668-1672$

Thes P M., Soumahoro J.A., Ackah J.A., Zihiri G.N. et Djama A.J. (2011) A pharmaceutical dermatological soap combining plant oils (MISCA) for the treatment of ringworm. Phytotherapie, 9 (6): 354-358.
Thes P. M., Zirihi G. N., Kra Adou K.M., Soumahoro I.A., Guede Guina F. (2006) Action du savon fait à partir des huiles de Mitracarpus Scaber (Rubiaceae)- » MISCA », Mareya Micrantha (Euphorbiaceae)- « G243 » et de Cassia Alata (Caesalpiniaceae) sur la croissance in vitro de Candida albicans.et Trichophyton mentagrophytes.

Traore F., Dosso M., Guede-Guina F. et Aka K.J. (2004) Effet myostimulant d'un extrait aqueux de Mareya micrantha (Euphorbiaceae) sur l'activité contractile intestinale de lapin. Ethnopharmacol, $33: 44-59$

Tsai C.S., Guede-Guina F., Smith M.O., Vangah M.M. et Ochillo R.F. (1995) Isolation of cholinergic active ingredients in aqueous extracts of Mareya micrantha using the longitudinal muscle of isolated guinea-pig ileum as a pharmacological activity marker. J of Ethnopharmacol, 45 : 215222. 